\title{
Profile Orientation and Slope Stability Analysis
}

\author{
Zhe-Ping Shen ${ }^{1,2}$ and Walter W. Chen ${ }^{2}$ \\ ${ }^{1}$ Department of Construction \& Spatial Design, Tungnan University, New Taipei 22202, Taiwan \\ ${ }^{2}$ Department of Civil Engineering, National Taipei University of Technology, Taipei 10608, Taiwan
}

Correspondence should be addressed to Walter W. Chen; waltchen@ntut.edu.tw

Received 15 January 2016; Revised 28 March 2016; Accepted 18 April 2016

Academic Editor: Eduardo Rodríguez-Tello

Copyright (c) 2016 Z.-P. Shen and W. W. Chen. This is an open access article distributed under the Creative Commons Attribution License, which permits unrestricted use, distribution, and reproduction in any medium, provided the original work is properly cited.

\begin{abstract}
This paper presents an analysis of soil slope stability using a terrestrial laser scanner, particle swarm optimization, and the force equilibrium method. The aim of this study was to demonstrate that a slope needed to be analyzed in many different directions in order to assess its stability conclusively, rather than using just one cross-sectional profile to represent the entire slope. To achieve this purpose, this study illustrates how a particle swarm optimization algorithm can be successfully incorporated into the analysis with slope stability analysis software, STABL. This study compares results obtained with those of previous studies and makes important observations.
\end{abstract}

\section{Introduction}

Rain-induced landslides are common phenomenon in subtropical Taiwan, which frequently experiences heavy rainfall from typhoons. The direction and mechanism of movement of an unstable slope are the subject of this study. Traditionally, the equilibrium condition of an earth slope is assessed by its Factor of Safety (FS), which is defined as the ratio of the total resisting force over the total driving forces, as shown in the following equation:

$$
\mathrm{FS}=\frac{F_{R}}{F_{D}},
$$

where $F_{R}$ is total resisting forces and $F_{D}$ is total driving forces.

The actual calculation of FS is a trial-and-error process because it depends on the selection of the sliding surface from many possible choices. Usually a fixed number of sliding surfaces are tested and the most critical sliding surface is the one that yields the smallest FS. A number of researches suggest that incorporating artificial intelligence algorithms such as the particle swarm optimization (PSO) into the computation could improve this practice. PSO is a computationally inexpensive algorithm developed by Kennedy and Eberhart $[1,2]$ that has roots in both artificial life and evolutionary computation. The following is one of its most original forms (with symbol changes):

$$
\begin{aligned}
& v=w v+c_{1} r_{1}(\text { pbest }-x)+c_{2} r_{2}(\text { gbest }-x), \\
& x=x+v,
\end{aligned}
$$

where $x$ is present location (randomly initialized), $v$ is particle velocity (randomly initialized), $w$ is inertia weight (added to the original PSO formula in [3]), $c_{1}$ is p_increment (=2 to overfly the target half the time [1]), $r_{1}$ is random number between 0 and $1, c_{2}$ is g-increment ( $=2$ to overfly the target half the time [1]), $r_{2}$ is random number between 0 and 1 , pbest is personally best position, and gbest is globally best position.

This paper presents a stability analysis of a slope near Taipei using PSO and FS. The slope was laser scanned to obtain accurate topographical profiles and analyzed from $0^{\circ}$ to $359^{\circ}$ at $1^{\circ}$ intervals. Since an ideal landslide study location is very difficult to come across and it may take years to complete a single study, the authors believe that this work represents a valuable case study for engineers interested in the multidirectional analysis of landslides using a swarm intelligence method. 


\section{Research Method}

The location of this study was near the Houshanyue hiking trail about $25 \mathrm{~km}$ from the city center of Taipei. The general area had a series of landslides after several typhoons within a few years. The subject of this study was one of the slopes affected by the landslides. The aerial photograph in Figure 1(a) shows the study location. Scanning the slope (marked by a red square) and the immediate area with a terrestrial laser scanner produced the point cloud model shown in Figure 1(b).

After the generation of the point cloud model, vegetation was removed digitally (using a software tool called RiScan Pro) to reveal the bare soils. Then, imaginary vertical cuts were made through the slope to create cross-sectional profiles for later slope stability analysis as shown in Figure 1(c). Three types of cuts were made. First, 45 north-south parallel crosssectional cuts ( $1 \mathrm{~m}$ apart) were made to study the variation of FS with profiles as illustrated in Figure 1(d). Next, 19 vertical cross-sectional cuts oriented $45^{\circ}$ to the north direction were made as shown in Figure 1(e) to study the variation of FS with profiles. The results of these two sets of analysis were presented in two conferences [4,5]. This study focuses on the last type of cut profile, which consisted of nonparallel cuts in directions ranging from $0^{\circ}$ to $359^{\circ}$ as shown in Figure 1(f). Consequently, this study examined and compared 360 possible profiles.

The research followed the steps shown in Figure 2. After downloading the point cloud data and the color panoramic photographs of the slope from the laser scanner to a desktop computer, the raw point cloud data and the panoramic photographs were registered together to create a point cloud model of the slope. The slope was cut from the highest point of the slope (lower left corner of the slope), and the slices were fanned out from $0^{\circ}$ to $359^{\circ}$. The vertical crosssectional profiles thus generated were fed into the standard slope stability analysis software, STABL (a 2D limiting equilibrium analysis program developed by Purdue University), to calculate the FS of each profile. Custom Fortran codes similar to that of [6] were used to read the output files of STABL and change the analysis parameters based on the PSO algorithm automatically. New input files were then generated by computer and fed into the STABL program again. The process continued itself until the FS values converged.

2.1. Simplified Bishop Method. The STABL program uses the method of slices for slope stability analysis [7]. For the method of slices (as illustrated in Figure 3), a slope is first dissected into many vertical slices and the force equilibrium and moment equilibrium are analyzed. Because there were more variables than equilibrium equations, we made assumptions to reduce the number of variables and solve for FS. Different methods of slices had different assumptions. For circular failure surfaces (the type of failure surfaces used in this study) in a soil slope, STABL recommended the simplified/modified Bishop method (using command CIRCL2) to achieve both the speed and accuracy [8]. Figure 3 shows a schematic diagram of the representative slice and the forces acting on the slice of the simplified Bishop method. There were four equilibrium assumptions:

(1) The overall moment equilibrium was satisfied.

(2) The slice moment equilibrium was not satisfied.

(3) The interslice vertical force equilibrium was satisfied.

(4) The interslice horizontal force equilibrium was not satisfied.

In other words, the simplified Bishop method assumed that the interslice forces were horizontal and that there were no shear forces. Below is the FS according to (1) and Figure 3 for the simplified Bishop method $[9,10]$ (with modification of symbols):

$$
\begin{aligned}
& \mathrm{FS}=\frac{\sum\left[c b_{i}+\left(W_{i}-u_{i} b_{i}\right) \tan \phi\right] / m_{\alpha}}{\sum W_{i} \sin \alpha_{i}}, \\
& m_{\alpha}=\cos \alpha_{i}\left\{1+\frac{\tan \alpha_{i} \tan \phi}{\mathrm{FS}}\right\}
\end{aligned}
$$

where $c$ is soil cohesion, $\phi$ is angle of friction, $b_{i}$ is width of soil slice, $l_{i}$ is width of soil slice along the sliding surface, $W_{i}$ is weight of soil slice, $T_{i}$ is resisting force of soil slice, $N_{i}$ is normal force of soil slice, $U_{i}$ is force of water, $u_{i}$ is pore pressure of water, $\alpha_{i}$ is slope angle of soil slice, and $E_{i}$ is interslice force.

2.2. Scripting Using PSO. Equations (3) show that the solution of FS is a highly complex function with no direct solutions. Since FS appears on both sides of the equations, the solution of FS requires an iterative approach. Substituting an initial assumed FS in (3) is necessary to compute a new FS. If the two FSs are different, then the initial FS is discarded. Equations (3) use the new FS to compute another FS value. The process will continue until both the assumed and the obtained FSs finally converge to the same value. Based on previous experience, the authors have found a technique to delegate this computation task to the STABL program and use the STABL program as a computation engine [6]. PSO, because of its simplicity, was ideal for this particular purpose. Neither did it have to know the inner working of STABL, nor did it have to know how to solve for the FS using the simplified Bishop method. All PSO needed to do was to control the execution of STABL and to adjust the parameters in the input files according to the results returned by the STABL program. In a sense, PSO functioned and acted similarly to a script, and the authors chose PSO for this study because of its relatively easy implementation and simple control.

\section{Computer Analysis}

The computer analysis in this study followed these steps:

(1) Create a point cloud model using RiScan Pro and raw scan data.

(2) Filter out vegetation and reveal bare soils underneath. 

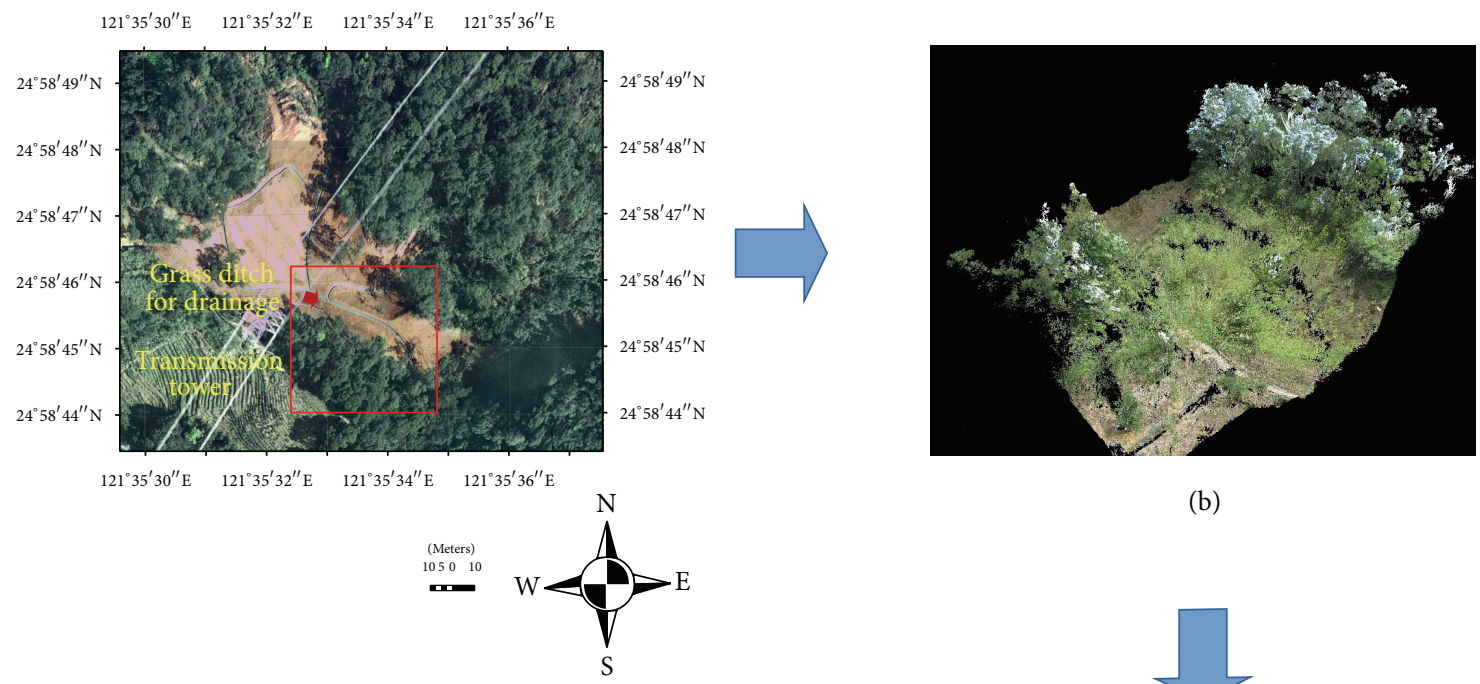

(b)

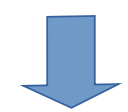

(a)

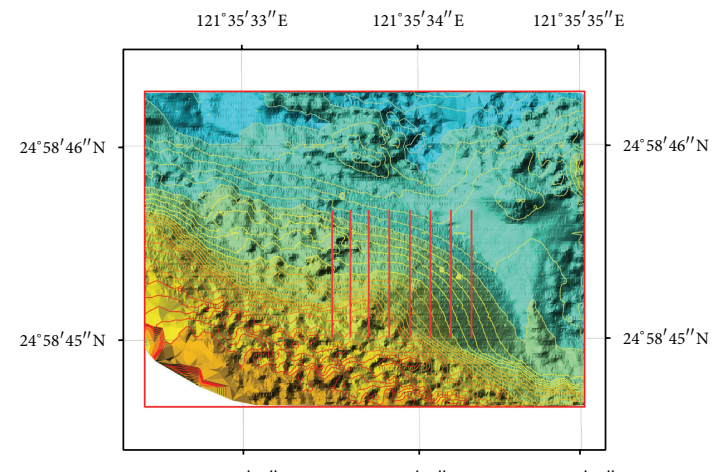

(d)
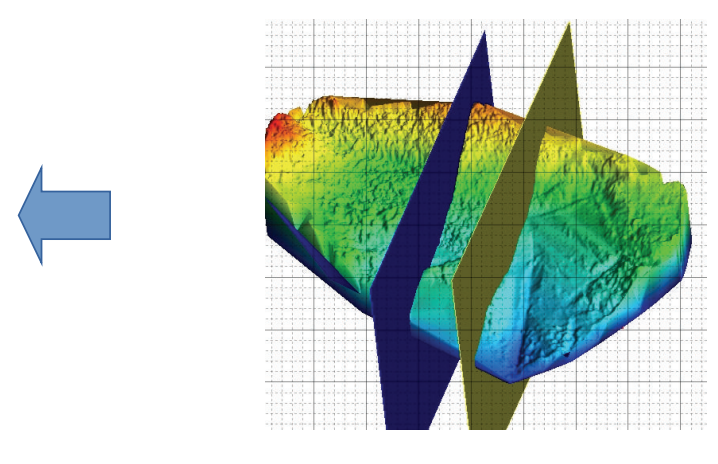

(c)

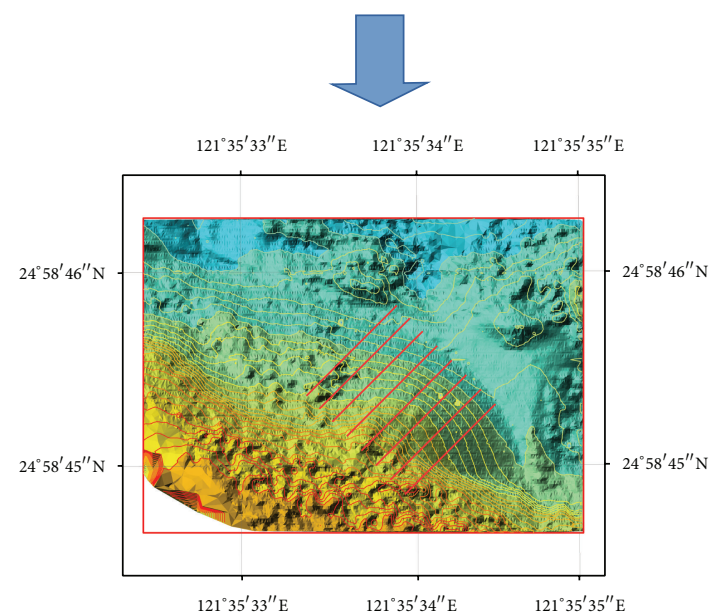

(e)

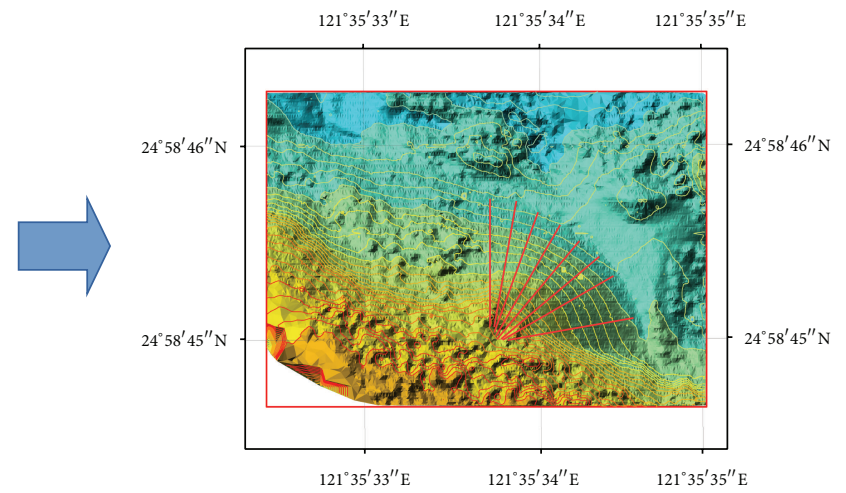

(f)

FIGURE 1: Research outline: (a) aerial photograph of the study location, (b) point cloud model of the slope, (c) imaginary cross-sectional cuts of the slope, (d) north-south parallel cuts of the slope, (e) $45^{\circ}$ parallel cuts of the slope, and (f) $0-359^{\circ}$ nonparallel cuts of the slope. 


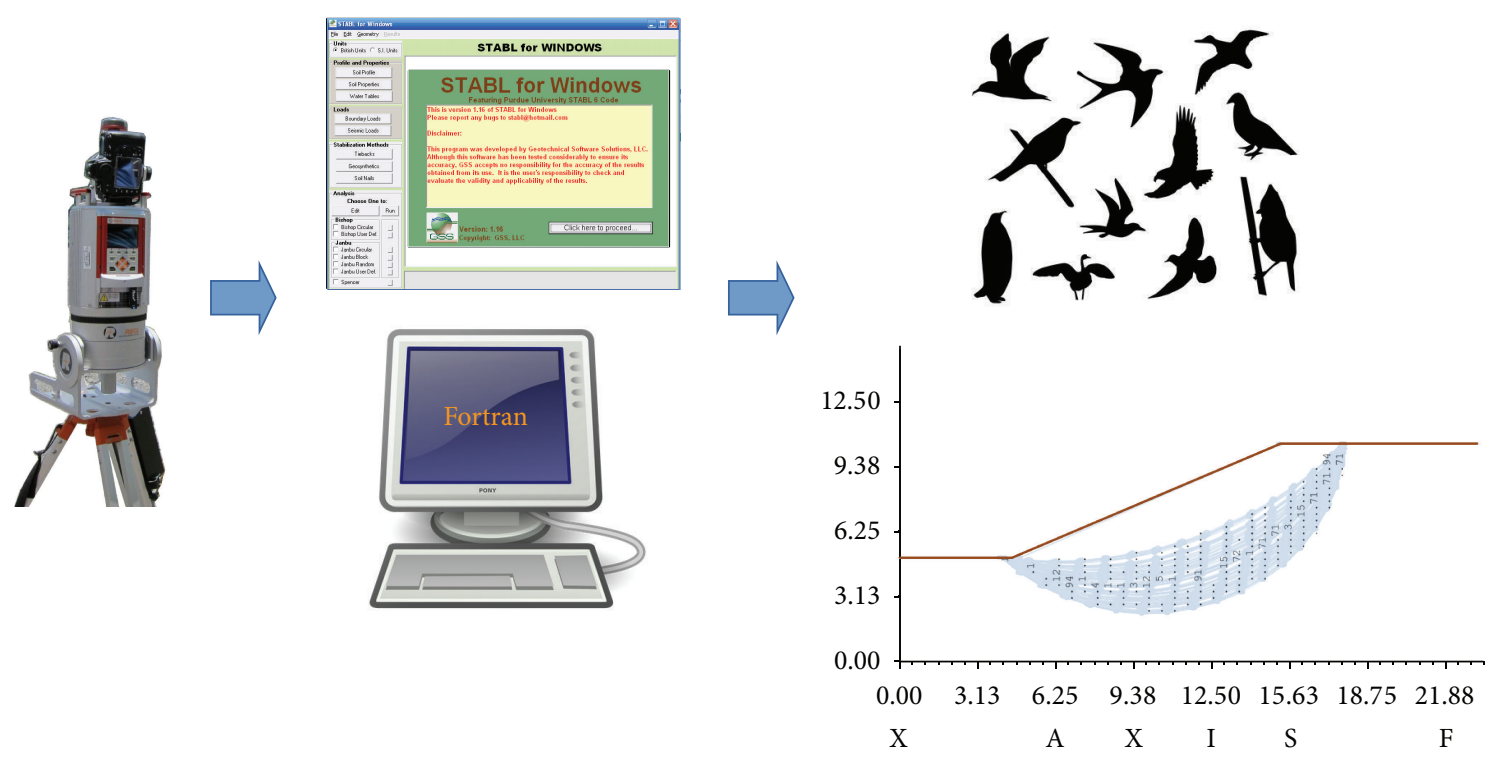

FIGURE 2: Illustration of research steps from laser scanning to slope stability analysis using STABL and Fortran and to incorporation of PSO algorithm.

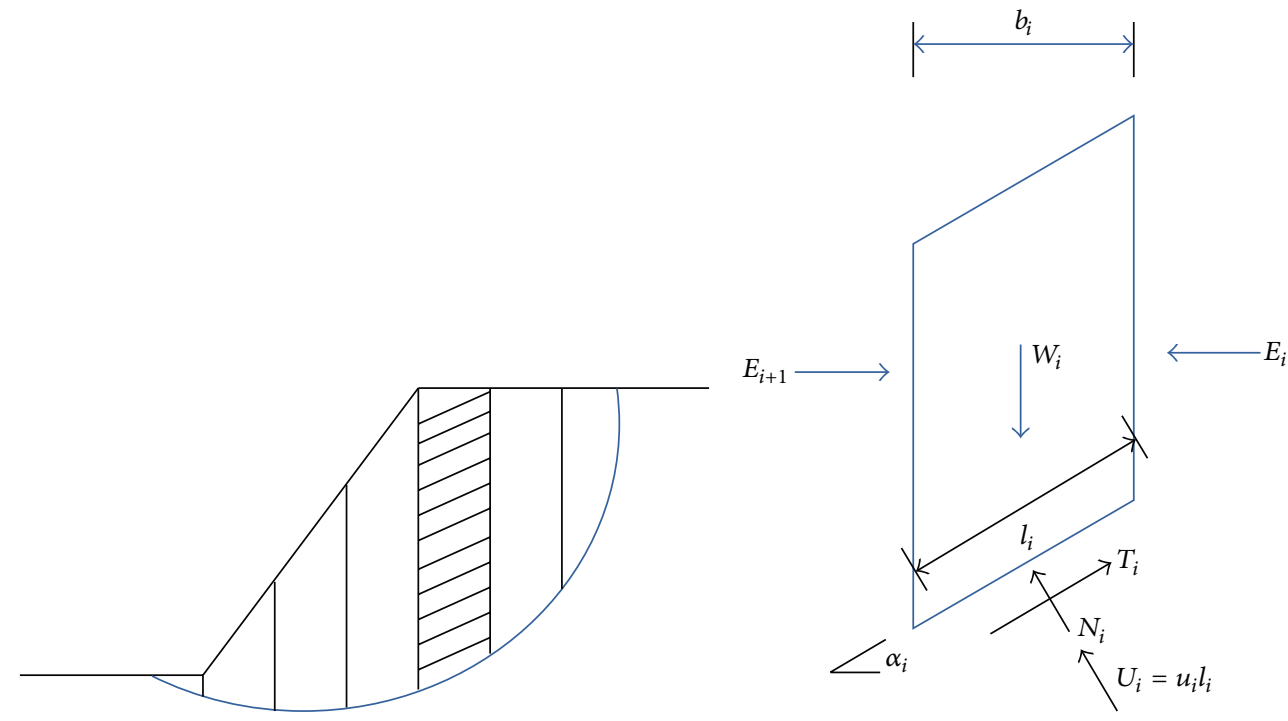

Figure 3: The schematic diagram of the simplified/modified Bishop method of slices.

(3) Use the triangulated irregular network (TIN) technique to create the ground surface representation of the slope (of bare soils).

(4) Select the zone/region of the slope for analysis.

(5) Choose the highest point of the slope.

(6) Using the highest point as the origin, use an imaginary vertical plane to dissect the slope from $0^{\circ}$ to $359^{\circ}$ in $1^{\circ}$ intervals. This is done programmatically.

(7) The cuts will intersect with the TIN surfaces and generate traces of cross-sectional profiles of the slope. This is also done programmatically.

(8) Because the lower left part of the terrain (southwest portion of the area in Figure 1) forms a hill, the sliding of the slope into the hill is not kinematically possible. Hence, only 180 profiles were generated out of 360 possible directions.

(9) Produce input files for the STABL program using the cross-sectional profiles and the soil parameters from a nearby boring test.

(10) The STABL program called by a scripting program processes the input files individually.

(11) The scripting program reads the output files (specifically the FS values in the output files) from the STABL program.

(12) The STABLE program processes the next set of input files based on the previous FS values and the PSO 


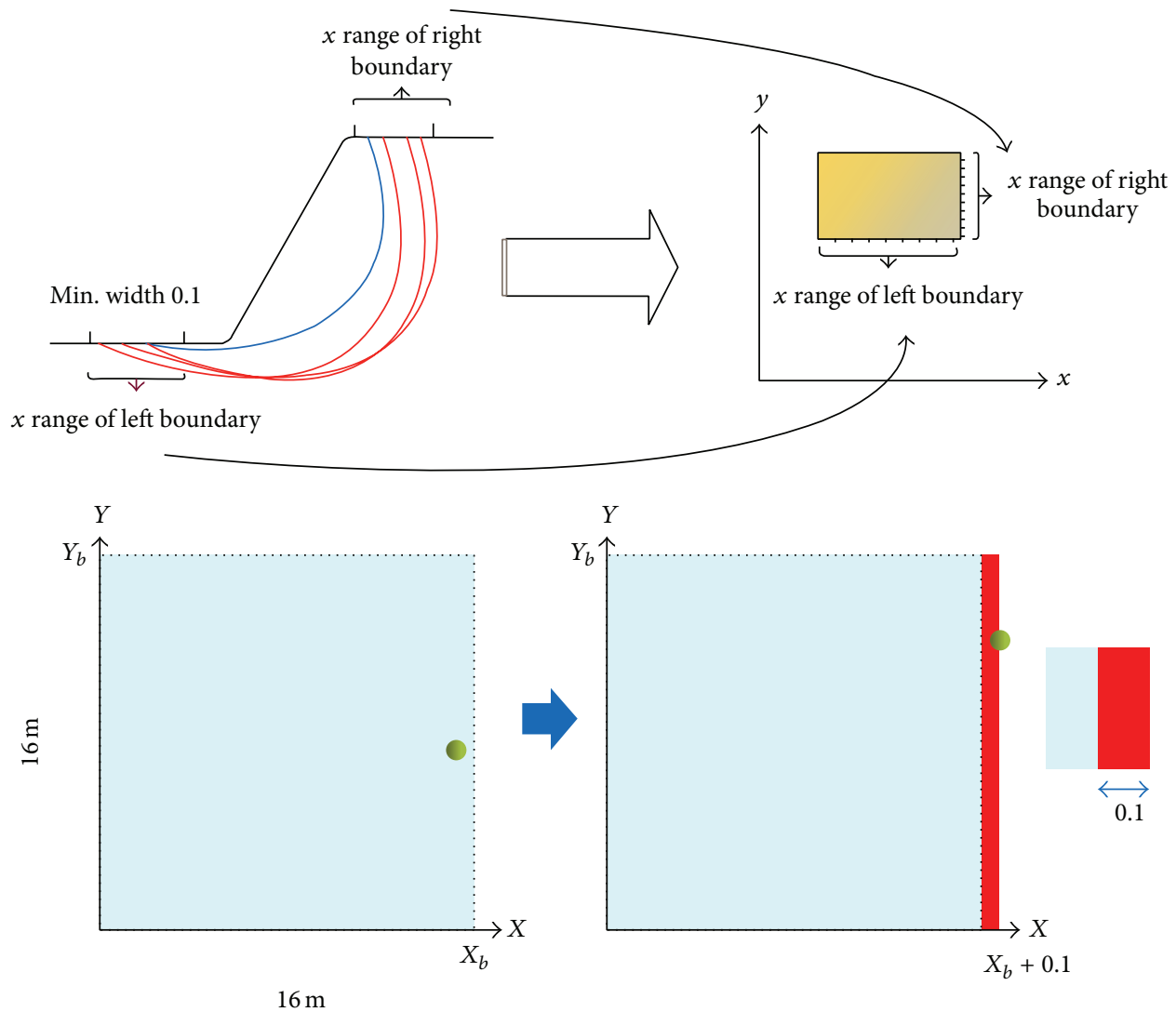

FIgURE 4: Relaxation of the boundary if a solution exists near the boundary.

algorithm, which generates a second set of output files. The process continues with successive sets of input files.

(13) The following parameters were used in the PSO script: $c_{1}=2, c_{2}=2, w=1$, and the number of particles $=$ 30. The number of iterations needed to converge for different profiles varies from 1 to 135 , with an average of 14.5 times.

(14) The scripting program stops when the FS values converge.

The STABL program selects random slip surfaces from the specified left and right horizontal ranges (as shown in Figure 4). Because the ranges were usually too wide to form the best slip surface and the corresponding minimum FS, adjusting the sizes of the left range and the right range at the same time was necessary. PSO optimizes the process by mapping the two ranges to a new $X-Y$ space. The $X$ coordinate of the new $X-Y$ space represents the left boundary of the left range, and the $Y$ coordinate represents the left boundary of the right range. In order to explore as many sliding surfaces as possible, the $X$ and $Y$ ranges were both set to be $16 \mathrm{~m}$, as shown in Figure 4 . The 16 by $16 X-Y$ space was defined as the search space, which was an imaginary space, created purely for PSO formulation. It is worth noting that within this imaginary space the object function (FS) is not an explicit continuous function. Rather, STABL needs to compute the FS value using (3) using an iterative approach. Also, note that STABL requires a minimum width of $0.1 \mathrm{~m}$ for both the left and the right ranges to work. Therefore, a solution $(x, y)$ in the new $X-Y$ space actually means the left range of $[x, x+0.1]$ and the right range of $[y, y+0.1]$. This small restriction does not usually interfere with the execution of the PSO and the STABL program. However, occasionally when the optimum solution is located near the boundary (as in Figure 4), the overall boundary of the new $X-Y$ space needs to be relaxed a little (by $0.1 \mathrm{~m}$ ) in order for STABL to function properly. An example is shown in Figure 5. Before the relaxation (a), PSO erroneously produced a higher FS than that of the initial trial. After relaxation (b), PSO was able to lower the FS as desired.

\section{Results and Discussion}

This study analyzed only the 180 directions that were kinematically possible, out of the 360 possible cut directions of the slope $\left(0^{\circ}-359^{\circ}\right)$. Two failed to produce any FS values with STABL $\left(81^{\circ}\right.$ and $\left.106^{\circ}\right)$ and therefore were not included in the subsequent analysis. Figure 6 summarizes the results. It shows that the minimum FS obtained in this study is 0.985 and the highest percentage of improvement by PSO is $20.05 \%$. Except for a few outliers, Figure 6 indicates that FS varies with the direction of the cross-sectional cut, apparently following a well-defined trend. The lowest FS values occurred in the $10^{\circ}$ 


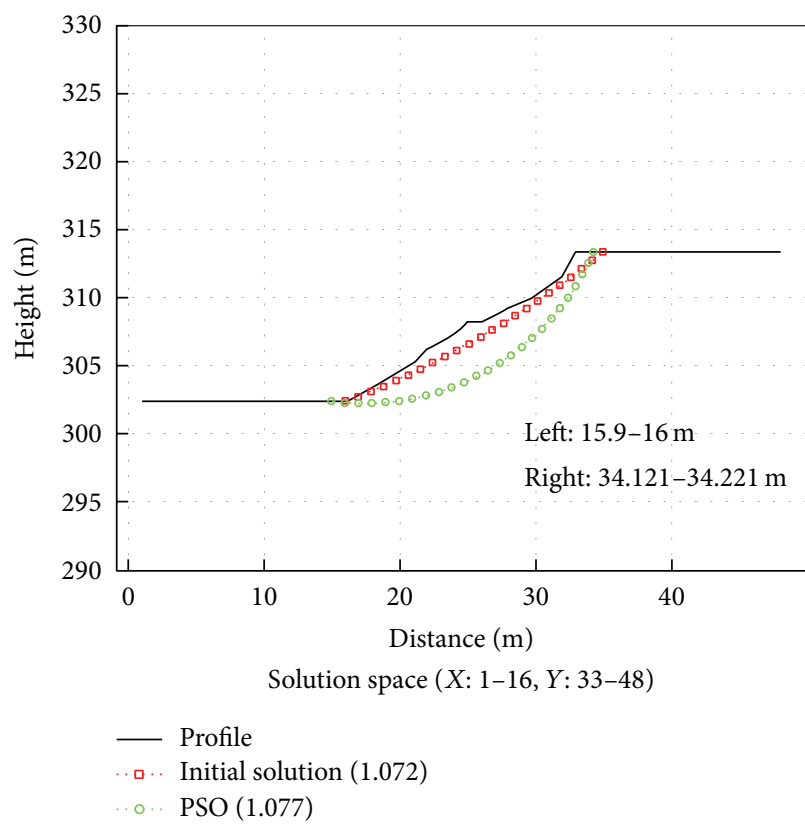

(a)

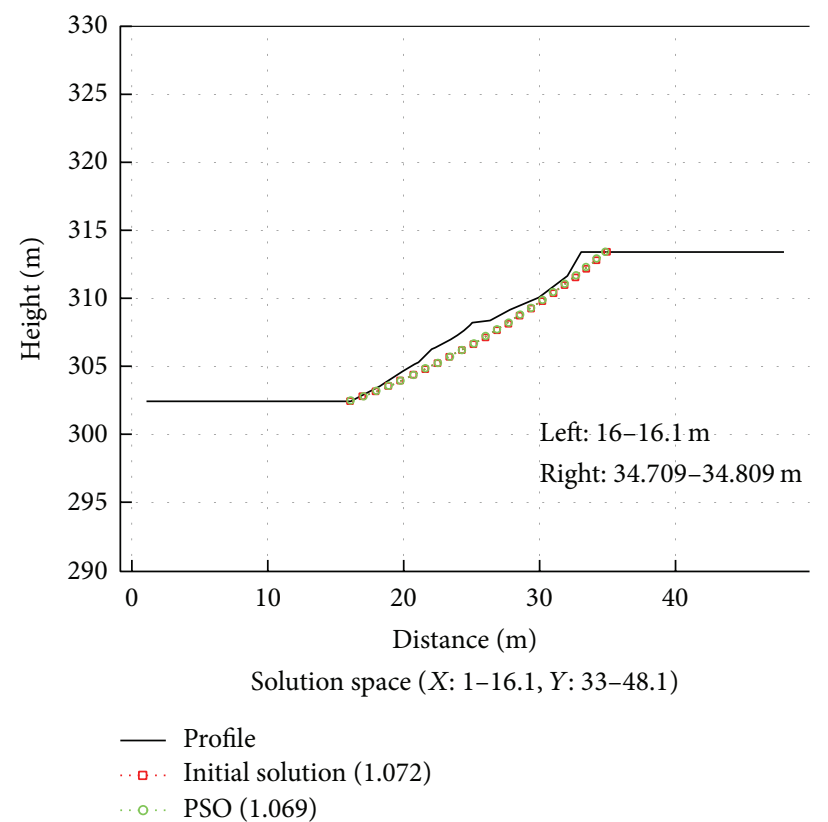

(b)

FIgURE 5: An example of relaxing boundaries by $0.1 \mathrm{~m}$ in order for STABL to function properly.

to $20^{\circ}$ range. Then, the FS value quickly increased away from this region and roughly reached the maximum at $111^{\circ}$ and $289^{\circ}$. No data points were available between $111^{\circ}$ and $289^{\circ}$ because it was kinematically impossible for the slope to move in the range of these directions (into the hill). It is clear from Figure 6 that FS is not a constant but rather a directionally dependent property of the slope. Therefore, using a "typical" 2D profile to represent the entire slope in traditional stability analysis is not very adequate.

4.1. Comparison with Previous Studies of the Same Site. Below are summaries of the analysis results from this study and two previous studies $[4,5]$ :

(1) $0^{\circ}$-degree parallel cuts [4]: the minimum FS among 45 profiles was 0.924 . The percentage of improvement against the original FS ranged from $0.91 \%$ to $10.25 \%$.

(2) $45^{\circ}$-degree parallel cuts [5]: the minimum FS among 19 profiles was 1.130 . The percentage of improvement against the original FS ranged from $0.16 \%$ to $11.11 \%$.

(3) $0^{\circ}-359^{\circ}$ degree nonparallel cuts: the minimum FS among 180 profiles was 0.985 . The percentage of improvement against the original FS ranged from $0.05 \%$ to $20.05 \%$.

Figure 7 below compares the results of the analysis from these three studies. The box-and-whisker plot used shows the minimum, the first quartile, the median, the third quartile, and the maximum of the data sets. Each study has two sets of FS data. They represent the FS values before and after applying the PSO algorithm to the computation. The two leftmost groups in Figure 7 are data from this study, while the middle two groups are from the north-south $\left(0^{\circ}\right.$

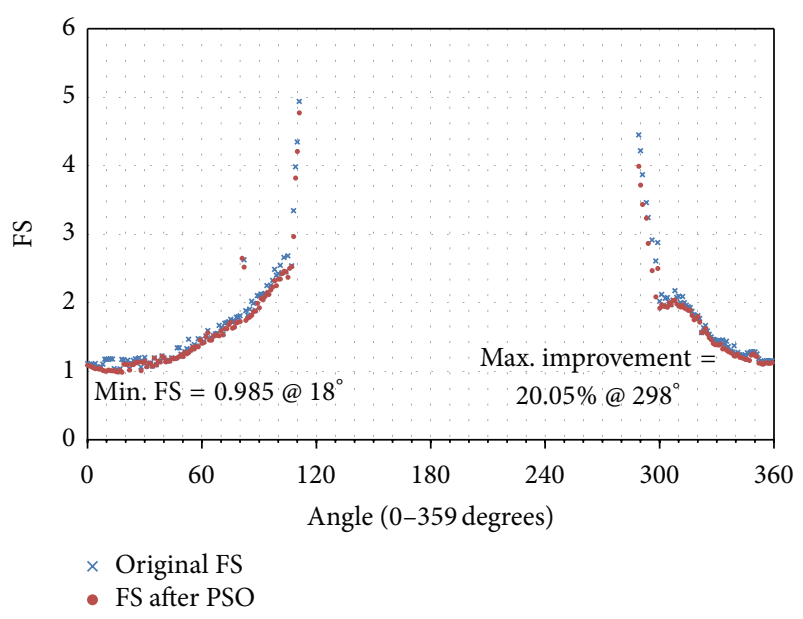

FIGURE 6: The results of 180 profiles analyzed in this study showing both the original FS values and the FS values after applying the PSO algorithm.

azimuth) profiles [4] and the two rightmost groups are from the $45^{\circ}$ azimuth cross-sectional profiles [5]. It is obvious from Figure 7 that this study is the most comprehensive one of the three studies. Not only does it provide the most data, but it also covers the widest range of possible FS values. Here are a number of observations:

(1) In all three studies, PSO improved (lowered) the FS. This is a successful demonstration of the applicability of PSO in the analysis of slope stability.

(2) 0.924 was the lowest FS obtained from the middle study, which represented north-south parallel 


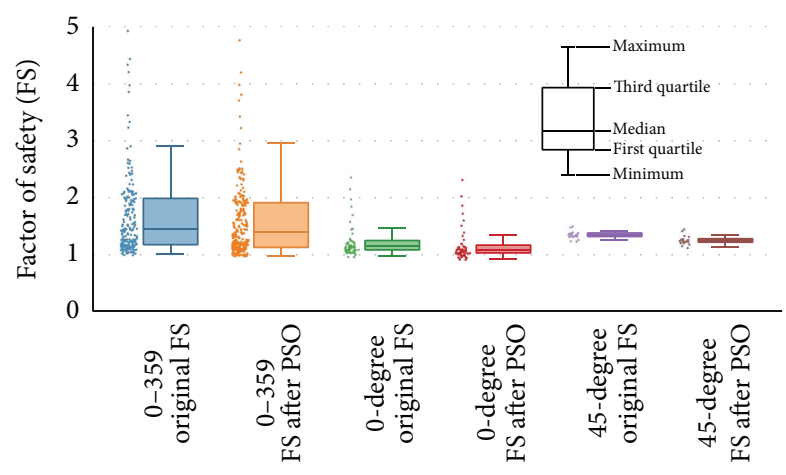

Types of analysis

FIGURE 7: The comparison of results from this study (leftmost two groups), 0 -degree cuts (middle two groups), and 45-degree cuts (rightmost two groups).

cross-sectional cuts. The lowest FS from the $0^{\circ}$ to $359^{\circ}$ study was a little higher (but not by much) at 0.985 . According to (1), a FS less than one means that the driving forces acting on the slope are greater than the forces resisting the sliding movement. Since in both cases the FS values ( 0.924 and 0.985$)$ were less than one, it indicates the instability of the analyzed slope.

(3) The rightmost two groups in Figure 7 (representing the study of $45^{\circ}$ parallel cuts) were the least satisfactory group in the comparison. The minimum FS obtained from this study was substantially higher than the minimums obtained from the other two studies.

(4) Figure 7 also shows that PSO improved the FS consistently, but the percentages of improvement resulting from the application of PSO were different for all three studies and varied from profile to profile. This is reasonable because the computation of FS is a highly complex iterative task (and nonlinear) performed by the STABL program. PSO was only used to optimize the boundary parameters sent to the STABL program.

(5) It is difficult to conclude from Figure 7 which type of analysis is most suitable for other slopes in the future. The $0^{\circ}$ to $359^{\circ}$ analysis was the most comprehensive, but the $0^{\circ}$ analysis resulted in a lower minimum FS. They all had their respective advantages and shortcomings. Moreover, slopes will have different ground surface profiles and soil parameters. Therefore, Figure 7 cannot provide any universal rules and apply to other slopes in general. We recommend analyzing all slopes in all possible manners using the automatic procedures shown in this study (and the two previous studies) in order to obtain conclusive results.

(6) The conclusion from combining all three studies is that the lowest FS of the analyzed slope is 0.924 . This is the result after analyzing $244(180+45+19)$ crosssectional profiles and should bear great significance to this slope. This FS value should also give the user a much higher level of confidence than that obtained from analyzing only one "typical" 2D slope profile.

4.2. Good Match with the Landslide Occurred. The analysis presented in this study was based on laser scanning of the target slope in June of 2011. The analysis showed that the slope was unstable. In August of 2012 after typhoon Saola, the slope indeed slid northward [11, 12], in the same general direction predicted by Figure 6. The sliding of the slope provides strong physical evidence in support of the above analysis.

4.3. Extension to More Sophisticated Evolutionary Algorithms. The technique presented in this study represents an ideal fit between slope stability analysis (STABL) and evolutionary computation (PSO). As mentioned in the research methods above, solving for FS was a complex task that required an iterative approach. However, treating the STABL program as a computation engine and a black box avoided the problem entirely. Using STABL rather than writing codes to calculate FS also made the analysis results more credible for comparison purposes. Using a combination of PSO and STABL also meant that there was no need to take the extra step to verify whether in-house codes performed just as well and yielded the same results as the standard STABL program. PSO did not need to know how STABL solved the FS. It only "told" STABL what to do in order to converge on the best results. Other, more sophisticated, artificial intelligence methods such as artificial neural networks (ANNs) are not applicable, because they require more interactions with the routines inside the STABL program, which is not possible since STABL is commercial software and not open source. As no other researcher has attempted this kind of separation of two tightly coupled components in slope stability analysis, FS calculation (STABL), and optimization (PSO), direct comparisons between the results of this study and other algorithms are not possible. However, other direct search optimization methods that might match the results and performance of PSO in this study could exist. Extending the current PSO + STABL approach to other evolutionary methods necessitates a new study.

4.4. Exhaustive Search. An exhaustive search examines every possible point in the search space. The authors conducted an exhaustive search on a fictitious soil slope problem (a commonly used slope by many researchers for theoretical studies and comparison) in a previous study [6]. 5000 grid points of the single 2D profile in [6] were analyzed. A similar exhaustive search was conducted in this study for the profile (among 180 profiles) with the minimum FS (0.985), which was oriented $18^{\circ}$ to the north direction. Figure 8 shows the 3D surface plot of the computed FS values, whereas Figure 9 shows the enlargement of the circled part of Figure 8 . The computation took around four hours on an Intel core i7 computer (including plotting), and the grid point was precise to one decimal place. From the PSO results mentioned earlier, it was determined that the minimum FS occurred at the point of $(15.900,34.870)$ in the imaginary $X-Y$ space (Figure 4$)$. Comparing this result with those shown in Figures 8 and 9, it 


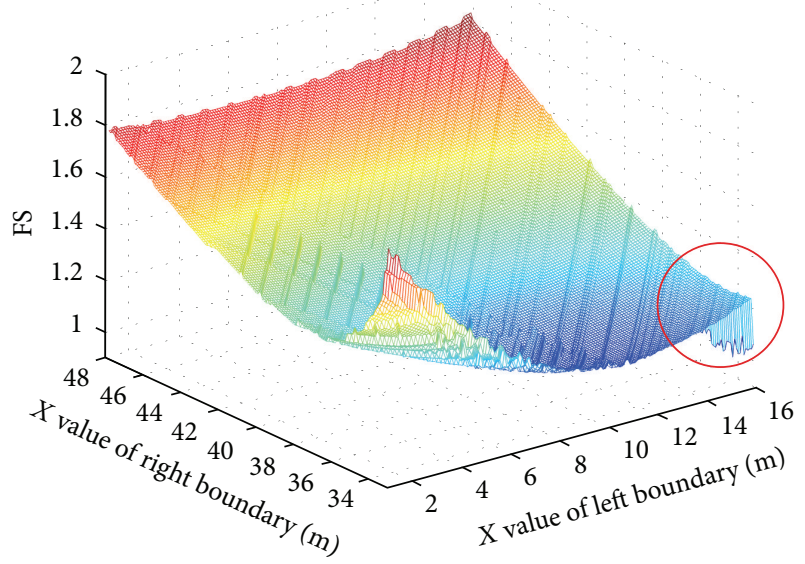

FIGURE 8: The solution space of the profile (among 180 profiles) with the minimum FS (0.985). This profile was oriented $18^{\circ}$ to the north direction.

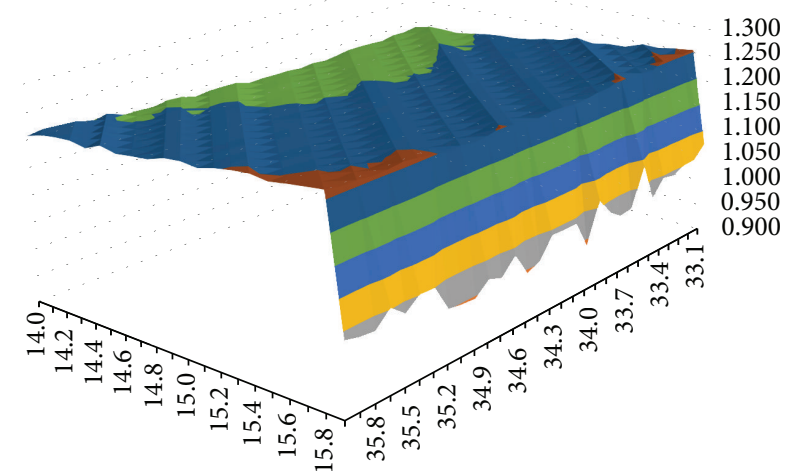

$$
\begin{aligned}
& \text { - 0.900-0.950 } \\
& \text { — } 1.100-1.150 \\
& \text { - 0.950-1.000 } \\
& \text { - 1.150-1.200 } \\
& \text { 1.000-1.050 } \\
& \text {. } 1.200-1.250 \\
& \text { - } 1.050-1.100 \\
& \text { - } 1.250-1.300
\end{aligned}
$$

FIGURE 9: The enlargement of the circled part of Figure 8, where the minimum FS solution is located.

is obvious that the exhaustive search only roughly determined the region where the minimum FS might occur (the frontfacing side of Figure 9). It did not pinpoint the exact location as the PSO method did. In order to achieve the same precise solution as PSO (from one-decimal place to three-decimal places), it would have required $100 * 100=10,000$ more times of computation than the current exhaustive search. The authors did not consider this additional computation to be beneficial for this study, because the reason for selecting PSO in the first place was to eliminate the huge time cost. If a three-decimal place exhaustive search had been used in this study, an analysis of a total of $16 \mathrm{~m} * 1000$ grids $/ \mathrm{m} *$ $16 \mathrm{~m} * 1000$ grids $/ \mathrm{m}=256,000,000$ grids would have been needed for a single profile. Since there were 180 profiles, roughly $256,000,000 * 180$ profiles $=46,080,000,000$ grid points would have been required. The STABL program needs calling for every grid point, with each call generating 100

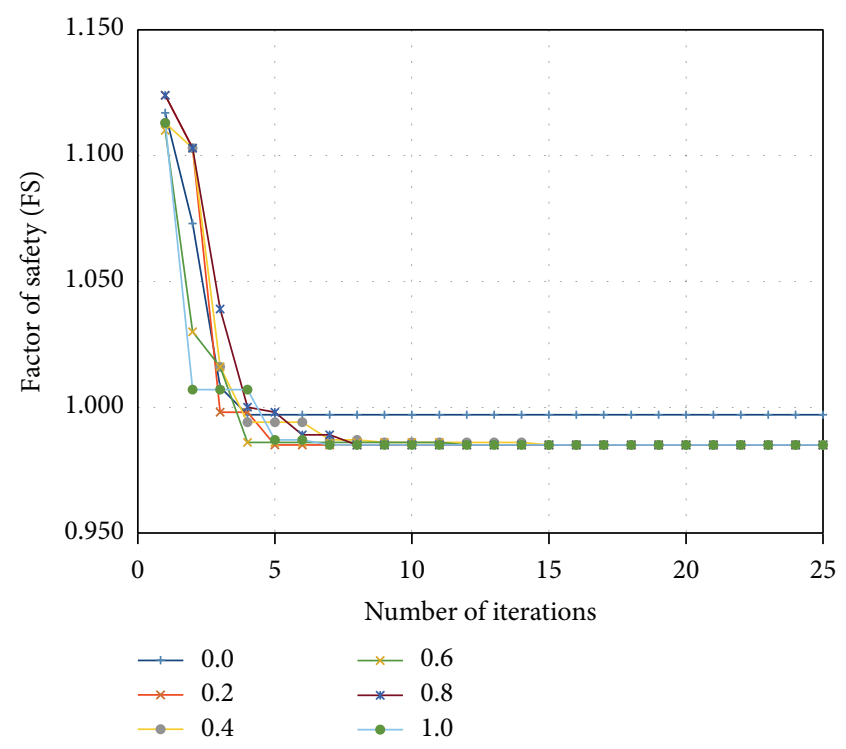

FIGURE 10: A set of convergence curves with different inertia weights $(w)$ for the profile with the minimum FS (0.985). The only curve that did not converge to the global minimum of 0.985 belongs to $w=0.0$ as expected from [6].

circular surfaces to compute the FS. Such large computation was difficult to justify and contradicted using PSO for its efficiency. Furthermore, the results from study [6] obtained by PSO were as good as the best results obtained by the exhaustive search. This important finding suggested that the addition of the exhaustive search was neither necessary nor beneficial for this study. It would not also help the understanding of the key points explored in this study, the purpose of which was to examine the use of PSO to accomplish an efficient and effective 3D slope stability analysis.

4.5. Randomness and Convergence. Typical PSO formulation has a number of parameters such as $c_{1}, c_{2}$, and $w$ that can be adjusted for better performance or avoidance of local minimums. However, the previous example soil slope study [6] showed that PSO converged quickly (usually only after dozens of iterations) when it was applied to the analysis of slope stability. Moreover, the selection of parameters (such as inertia weight) and random seeds had almost no influence on either the global minimum discovery or the system performance, as long as the inertia weight chosen was not close to zero. For verification purposes, a convergence test of different inertia weights (for $w=0.0,0.2,0.4,0.6,0.8$, and 1.0) was repeated for the profile with the minimum FS of 0.985 in this study (as shown in Figure 10). The result is very similar to that conducted on a fictitious example soil slope in [6]. As can be seen from Figure 10, all curves converged quickly, and the only curve that did not converge to the global minimum of 0.985 belongs to $w=0.0$, as expected from [6]. Since the inertia weight had only negligible influence on the convergence behavior (as long as the inertia weight was not zero), the authors decided to use $w=1$ and the number of particles equal to 30 in the analysis of the remaining the profiles. 


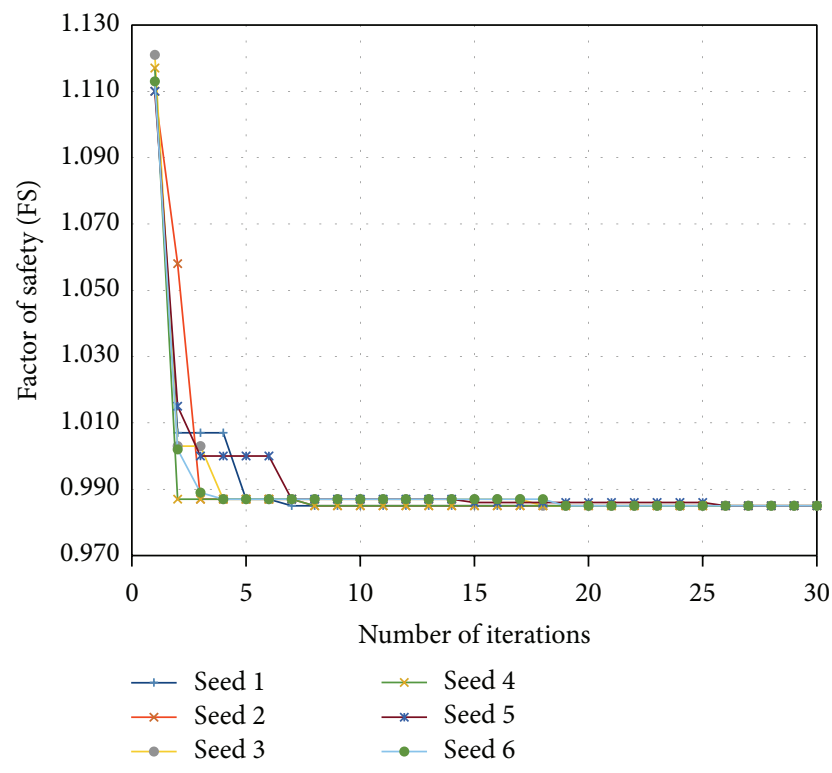

FIGURE 11: A set of convergence curves with the same inertia weight $(w=1.0)$ but different initial random seeds for the profile with the minimum FS (0.985).

To test different initial random seeds, Figure 11 shows the results of six different runs (only six are shown in the figure to avoid clutter). Again, all curves converged quickly to the global minimum (less than 26 times of iterations). It verifies the conclusion of [6] that the influence of random seed was also negligible. Looking at Figure 11 closely together with Figures 8 and 9, the reason behind this result is clear. Although the computation of FS using the modified Bishop method (equations (3)) was an iterative procedure and highly complex, the solution of FS shown in Figure 8 represents a relatively simple surface. The minimum of this surface was located in the thin region near the boundary and unlikely missed, no matter what the initial random seed was.

The overall performance of the approach outlined in this paper is satisfactory. More importantly, PSO improved (lowered) the FS in every case. Moreover, the results obtained were as precise as they could be. This is because STABL only outputs FS to three-decimal places. Therefore, any further tuning of the PSO parameters will not yield a more precise result. For example, suppose that the minimum FS is 0.985 , and it corresponds to a point $(15.900,34.870)$ in the imaginary $X-Y$ space. If there existed a point close to $(15.900,34.870)$ with an even lower FS, it will not be discovered because STABL will return the same FS of 0.985 for the new point. Since the FSs are the same, the input parameters will not be adjusted and PSO will not attempt further optimization. This is a shortcoming of using STABL as a block box in computation.

4.6. Other Related Works. PSO is a powerful yet simple optimization technique that has been applied to many different fields to solve many different types of problems, including the stability analysis of earth slopes. For earth slopes, PSO was primarily used to compute the minimum FS and to determine the most critical sliding surface [13-20]. In addition to theoretical framework development, a number of real-world engineering case studies were also available. For example, Khajehzadeh et al. [16] back-calculated the FS of a landslide in Ulu Klang, Malaysia; Wang et al. [21] used PSO to assess the stability of the valley shoulder deposits on the left bank of the Xiluodu Hydropower Station in China; and Li et al. [22] applied PSO to the reinforcement design of the Zhongjiawu high cut slope of the Three Gorges. However, most of these PSO-related studies were based on 2D profiles, while none used laser scanning and STABL in the analysis. Of these studies, only one considered 3D analysis of slope stability by means of a general rotating ellipsoid shape [17]. Nevertheless, the method was entirely different from the approach taken in this study, which analyzed the target slope in directions ranging from $0^{\circ}$ to $359^{\circ}$. Originally, this study only preliminarily analyzed the slope using STABL in [11]. Later, the analysis in [12] incorporated PSO with manually generated profiles (instead of automatically created profiles). The total combined horizontal range (left and right) of the slope in these studies was about $20 \mathrm{~m}$, which was less than the current study. Because of the differences in analysis, the FS values of these studies were different from the FS values in this study, in which a dissection program cut the slope automatically and the horizontal ranges extended.

\section{Summary and Conclusions}

Landslides are a major problem affecting millions of people around the world. The goal of this study was to understand how using PSO could improve the analysis of questionable slopes before a landslide took place. State-of-the-art equipment (a terrestrial laser scanner) was used to generate 3D ground surfaces, and industry standard application software (STABL) was used in the analysis of slope stability. The authors also developed in-house custom programs to cut the slope and generate profiles in many different directions. The main use of the PSO algorithm was to optimize the parameters related to the boundaries of the slope where sliding surfaces initiated and ended. Using the STABL program as a computation engine, the proposed system was able to read output files and generate new input files automatically. With this analysis capability, it was then possible to analyze the slope in directions ranging from $0^{\circ}$ to $359^{\circ}$. This study proved that a complete analysis of a slope could be performed automatically. As such, it removed the limitations of treating the slope as a $2 \mathrm{D}$ object and analyzing only one crosssectional profile to represent the entire slope. The results showed that FS was not a constant but rather a directionally dependent property of the slope and that more accurate information regarding the state of the slope resulted from thoroughly analyzing the slope in many directions. Armed with this information, it is less likely that engineers will ignore unstable slopes in the future. This study also demonstrated that the PSO algorithm was highly applicable in the analysis of slope stability. Summarizing this research and two previous studies of the same site, the lowest FS of the analyzed slope was 0.924 . This was the result after analyzing $244(180+45+$ 19) cross-sectional profiles. Therefore, this value should have 
great significance and should give the engineer a much higher level of confidence.

\section{Competing Interests}

The authors declare that they have no competing interests.

\section{Acknowledgments}

This study was partially supported by Grant numbers NSC 102-2221-E-027-077 and MOST 103-2221-E-027-052 from the National Science Council and the Ministry of Science and Technology of Taiwan. The financial support is highly appreciated.

\section{References}

[1] J. Kennedy and R. Eberhart, "Particle swarm optimization," in Proceedings of the IEEE International Conference on Neural Networks, pp. 1942-1948, IEEE Service Center, Perth, Australia, 1995.

[2] R. Eberhart and J. Kennedy, "A new optimizer using particle swarm theory," in Proceedings of the 6th International Symposium on Micro Machine and Human Science (MHS '95), pp. 3943, Nagoya, Japan, October 1995.

[3] Y. Shi and R. Eberhart, "Modified particle swarm optimizer," in Proceedings of the IEEE International Conference on World Congress on Computational Intelligence, pp. 69-73, Anchorage, Alaska, USA, May 1998.

[4] Z.-P. Shen and W. W. Chen, "Slope stability analysis using multiple parallel profiles," in Proceedings of the 11th International Conference on Natural Computation (ICNC '15) and the 12th International Conference on Fuzzy Systems and Knowledge Discovery (FSKD '15), Zhangjiajie, China, August 2015.

[5] Z.-P. Shen and W. W. Chen, "Directional analysis of slope stability using a real example," in Proceedings of the 6th International Conference on Swarm Intelligence (ICSI '15), Beijing, China, June 2015.

[6] W. W. Chen, Z.-P. Shen, J.-A. Wang, and F. Tsai, "Scripting STABL with PSO for analysis of slope stability," Neurocomputing, vol. 148, pp. 167-174, 2015.

[7] R. A. Siegel, STABL User Manual, Purdue University, West Lafayette, Ind, USA, 1975.

[8] E. Achilleos, User Guide for PC STABL 5M, Purdue University, West Lafayette, Indiana, 1988.

[9] R. Salgado, The Engineering of Foundations, McGraw-Hill International Edition, McGraw-Hill, New York, NY, USA, 2008.

[10] W.-T. Wu, Slope Stability Analysis Methods and Applications, 1980 (Chinese).

[11] C.-K. Hsu and W. W. Chen, "New landslide at Mt. Houshanyue hiking trail," in Proceedings of the International Symposium on Remote Sensing (ISRS '13), Tokyo, Japan, May 2013.

[12] Z.-P. Shen, C.-K. Hsu, and W. W. Chen, "Slope stability analysis of houshanyue landslide using STABL and PSO," in Proceedings of the 34th Asian Conference on Remote Sensing (ACRS '13), Bali, Indonesia, October 2013.

[13] L. Li and X.-S. Chu, "An improved particle swarm optimization algorithm with harmony strategy for the location of critical slip surface of slopes," China Ocean Engineering, vol. 25, no. 2, pp. 357-364, 2011.
[14] M. Khajehzadeh, M. R. Taha, and A. El-Shafie, "Reliability analysis of earth slopes using hybrid chaotic particle swarm optimization," Journal of Central South University of Technology, vol. 18, no. 5, pp. 1626-1637, 2011.

[15] H. Li, H. Zhong, Z. Yan, and X. Zhang, "Particle swarm optimization algorithm coupled with finite element limit equilibrium method for geotechnical practices," Mathematical Problems in Engineering, vol. 2012, Article ID 498690, 14 pages, 2012.

[16] M. Khajehzadeh, M. R. Taha, A. El-Shafie, and M. Eslami, "Stability assessment of earth slope using modified particle swarm optimization," Journal of the Chinese Institute of Engineers, vol. 37, no. 1, pp. 79-87, 2014.

[17] R. Kalatehjari, A. S. A Rashid, N. Ali, and M. Hajihassani, "The contribution of particle swarm optimization to threedimensional slope stability analysis," The Scientific World Journal, vol. 2014, Article ID 973093, 12 pages, 2014.

[18] S. Xiao, K. Li, X. Ding, and T. Liu, "Numerical computation of homogeneous slope stability," Computational Intelligence and Neuroscience, vol. 2015, Article ID 802835, 10 pages, 2015.

[19] B. Li, D. Li, Z. Zhang, S. Yang, and F. Wang, "Slope stability analysis based on quantum-behaved particle swarm optimization and least squares support vector machine," Applied Mathematical Modelling, vol. 39, no. 17, pp. 5253-5264, 2015.

[20] B. Gordan, D. J. Armaghani, M. Hajihassani, and M. Monjezi, "Prediction of seismic slope stability through combination of particle swarm optimization and neural network," Engineering with Computers, vol. 32, no. 1, pp. 85-97, 2015.

[21] S. N. Wang, C. Shi, Y. L. Zhang, and K. H. Chen, "Numerical limit equilibrium analysis method of slope stability based on particle swarm optimization," Applied Mechanics and Materials, vol. 353-356, pp. 247-251, 2013.

[22] S. Li, H. Gao, D. Xu, and F. Meng, "Comprehensive determination of reinforcement parameters for high cut slope based on intelligent optimization and numerical analysis," Journal of Earth Science, vol. 23, no. 2, pp. 233-242, 2012. 

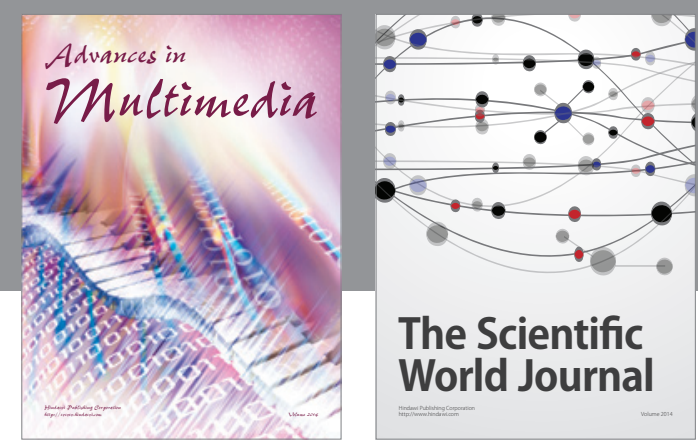

The Scientific World Journal
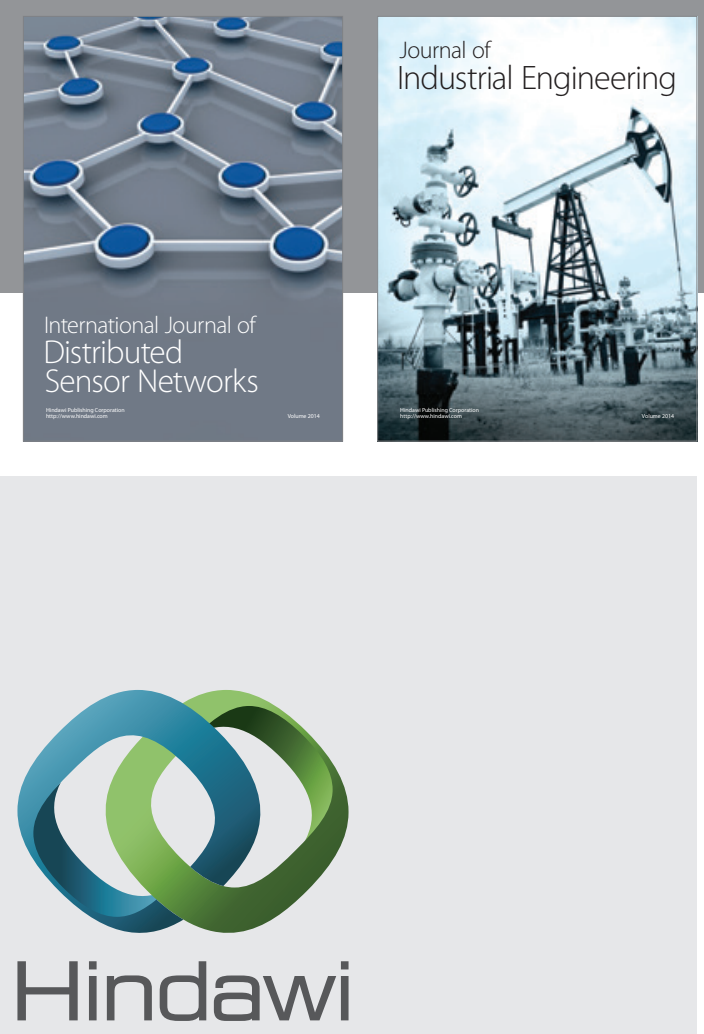

Submit your manuscripts at

http://www.hindawi.com

\section{Computer Networks} and Communications
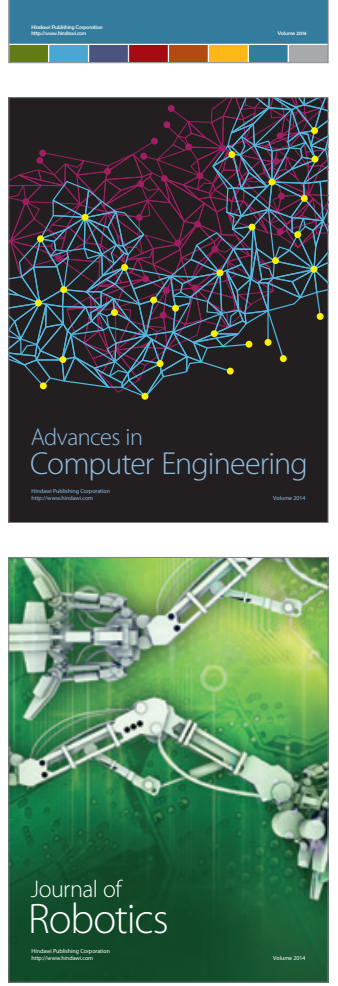
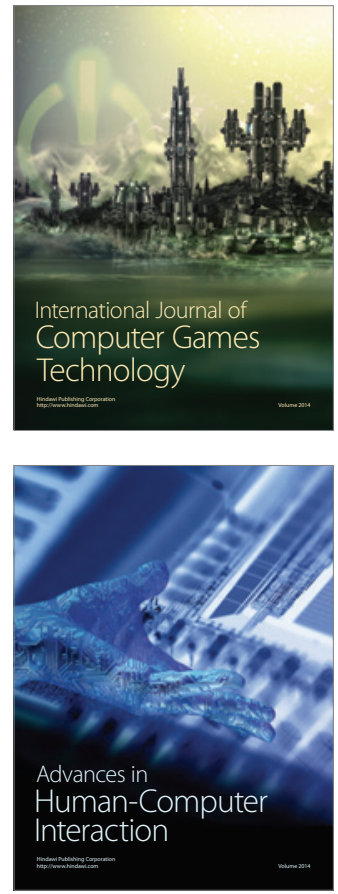
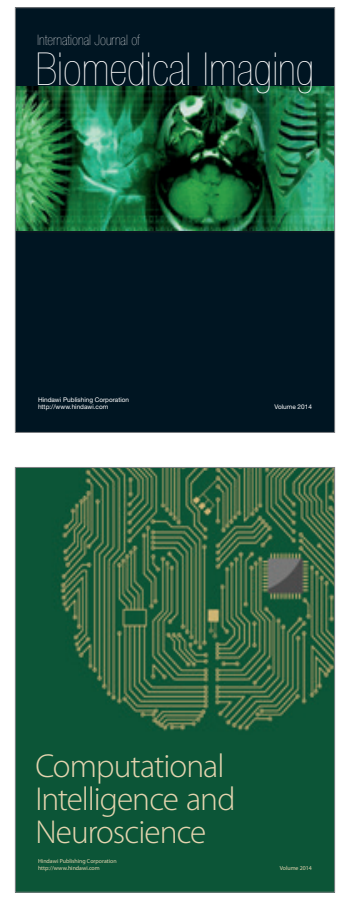
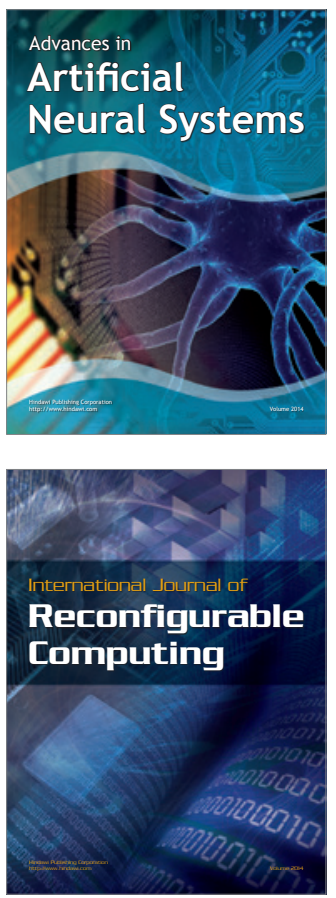
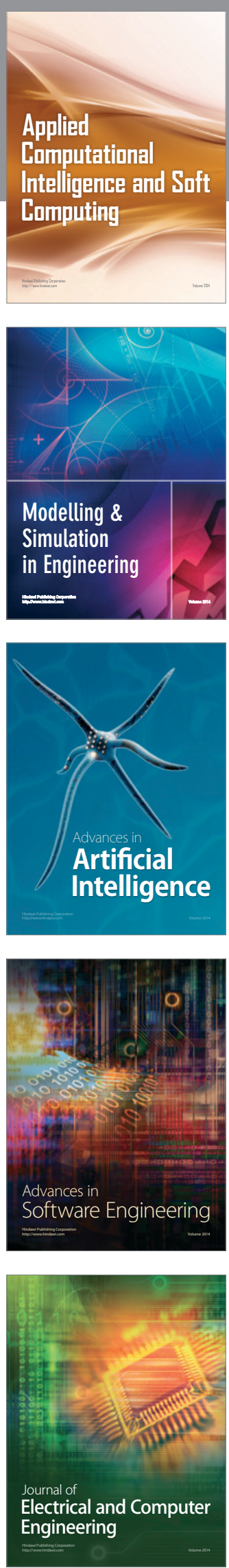